\title{
RESEARCHES ON THE ACCURACY OF THE DCMT CIRCLE READING SYSTEM
}

\author{
$\mathrm{ZHU} \mathrm{ZI}$ \\ Shaanxi Astronomical Observatory \\ Chinese Acadeny of Sciences \\ P.O.Box 18, Lintong, Shaanxi \\ P.R.China
}

SUMMARY: The declinations of stars are determined by the circle micrometers on the meridian circle. The determined accuracy of declinations depends on the systematic accuracy of the micrometers. The DCMT (Danish Chinese Meridian Telescope) adopts photoelectric scanning micrometers as the circle reading system. It contains 6 micrometers forming 3 pairs to measure the circle posistion simultaneously. The repeatability of the circle reading and systematic errors of micrometers are discussed in this paper.

To measure the repeatability, we fix the circle and the 6 micrometers scan the division lines simultaneously. The mean square error of the position for a line derived from the repeated scans of a micrometer is the repeatability of the reading. The position of a line is calculated from the readout values in a profile by means of the linear least-squares fit. It is measured that the repeatability for all six micrometers is better than $0 .{ }^{\prime} 03$ and the average value is $0 . .^{\prime} 022$.

The systematic error of a micrometer means that the scamning distance is not proportional to the angle distance on the circle which is scanned. It is caused by many parameters of the micrometer. We have found that the systematic error strongly depends on the adjustment status of the micormeter.

The systematic error can be described with a density function of the distribution of errors $g(x)$, where $x \in[0 . T], T$ is the maximum of scanning. If the positions $r_{1}, r_{2}\left(r_{1}>r_{2}\right)$ of two arljacent lines are measured, we can get the corrections to the systematic errors. The accuracy of the position measurement is greatly improved after the correction. The mean error of the distance measurements between two lines for single micrometer reaches $0 .{ }^{\prime \prime} 061$ and the accuracy for every micrometer is similar, while this error is more high and differnt for distinct micrometers without correction. The single measurement of single line has a accuracy of $0 .{ }^{\prime} 043$ with correction.

\section{REFERENCES}

Einicke,O.H. et al. 1971, Astro. \& Astrophys., 10

Li Huanxin, 1990, Pulbl. of CSAO, Vol.13, No.1.

Li Huanxin, 1991, Publ. of CSAO, Vol.14, No.2.

Xu Linsheng, Li Zhigang, 1991, Publ. of CSAO, Vol.14, No.1.

Zhı Zi, Liu Shaomin, 1991, Publ. of CSAO, Vol.14, No.1. 